(C) 2010, IEEE. Reprinted, with permission, from McDonald, G, Christian, J and Hodgkinson, T, Optical soliton pulses with relativistic characteristics, Advanced Optoelectronics and Lasers (CAOL), 2010 International Conference, 2010.

This material is posted here with permission of the IEEE. Such permission of the IEEE does not in any way imply IEEE endorsement of any of the University of Salford's products or services. Internal or personal use of this material is permitted. However, permission to reprint/republish this material for advertising or promotional purposes or for creating new collective works for resale or redistribution must be obtained from the IEEE by writing to pubs-permissions@ieee.org. 


\title{
Optical soliton pulses with relativistic characteristics
}

\author{
G. S. McDonald, J. M. Christian, T. F. Hodgkinson \\ Materials \& Physics Research Centre, University of Salford, U.K.
}

\begin{abstract}
The slowly varying envelope approximation (SVEA) and the ensuing Galilean boost to a local time frame are near-universal features of conventional scalar pulse models. They have enjoyed unbridled longevity in the literature over the past forty years for two main reasons. Firstly, they often provide an adequate description of the phenomena being observed. Secondly, a large body of knowledge exists on how to solve the resultant parabolic governing equations. In this Invited presentation, we consider the consequences of relaxing both the SVEA and the Galilean boost. These two artefacts are tightly connected, and in ways that are not always obvious. We will give a complete overview of our recent discoveries concerning a generalized nonlinear Helmholtz equation that describes optical pulses in the laboratory reference frame.
\end{abstract}

\section{INTRODUCTION}

It can be safely said that optical soliton pulses are one of the most thoroughly investigated and well-understood phenomena in nonlinear photonics. Since the seminal works of Hasegawa and Tappert [1], and later the experiments of Mollenauer et al. [2], the cornerstone of many investigations has been the slowly-varying envelope approximation (SVEA). The SVEA, in combination with a subsequent Galilean boost to a local time frame, tends to reduce the complexity of the longitudinal (spatial) part of wave operator, with temporal effects (such as higher-order dispersion and Raman scattering) left unchanged. While this approach has some clear-cut advantages [by replacing the elliptic (or hyperbolic) governing equation with a parabolic one], there are some physical effects that fall outside its remit. One such effect is spatial dispersion [3], recently discussed by Biancalana and Creatore in the context of pulse envelopes in semiconductor planar waveguides [4].

Here, we report our recent discoveries surrounding a very general Helmholtz model of scalar optical pulses. By deploying similar methods to those used over the past ten years to study nonlinear beams, we have been able to derive several classes of exact analytical soliton solution to a new governing equation. Our results are fundamentally different from those already published [4] because of the frame of reference in which we analyze the pulses. They have a simple physical interpretation, and some surprising connections to special relativity have been uncovered.

\section{Helmholtz Pulse Model}

We begin by considering a scalar electric field $E(t, z)$ that is traveling down the longitudinal axis $z$ of a waveguide,

$$
\begin{aligned}
E(t, z)=A(t, z) \exp [ & \left.i\left(k_{0} z-\omega_{0} t\right)\right] \\
+ & A^{*}(t, z) \exp \left[-i\left(k_{0} z-\omega_{0} t\right)\right],
\end{aligned}
$$

where $t$ is the time coordinate. Here, $A(t, z)$ is the envelope that modulates a carrier wave with optical frequency $\omega_{0}$ and propagation constant $k_{0}=n_{0} \omega_{0} / c$, where $n_{0}$ is the linear refractive index of the core medium at $\omega_{0}$ and $c$ is the vacuum speed of light. The transverse spatial variation of the electric field is controlled by the structure of the waveguide itself. By substituting Eq. (1) into the corresponding Maxwell equations and Fourier transforming to the temporal frequency domain, it can be shown that [5]

$$
\frac{\partial^{2} \tilde{A}}{\partial z^{2}}+i 2 k_{0} \frac{\partial \tilde{A}}{\partial z}+\left(k^{2}-k_{0}^{2}\right) \tilde{A}=0,
$$

where $\tilde{A} \equiv \tilde{A}(\omega, z)$ denotes the Fourier transform of the pulse envelope. The parameter $k^{2}$ that appears in Eq. (2) is the mode eigenvalue - it is obtained by solving Maxwell equations for the transverse part of the confined field. The factor $\left(k^{2}-k_{0}^{2}\right)$ is often approximated by $2 k_{0}\left(k-k_{0}\right)$, and the remaining linear term $k \equiv k(\omega)$ is expanded around $\omega_{0}$ according to

$$
k(\omega)-k_{0}=\sum_{j=1}^{\infty} \frac{k_{j}}{j !}\left(\omega-\omega_{0}\right)^{j}+\Delta k_{\mathrm{NL}},
$$

where $k_{0} \equiv k\left(\omega_{0}\right)$, and $k_{j} \equiv\left(\partial^{j} k / \partial \omega^{j}\right)_{\omega 0}$ for $j=1,2,3, \ldots$ The last term on the right-hand side of Eq. (3) is the nonlinear correction to $k$ is taken to be $\Delta k_{\mathrm{NL}}=n_{2} I \omega_{0} / c$, where $n_{2}$ is the Kerr coefficient and $I$ is the intensity. The summation in Eq. (3) is truncated by assuming that terms beyond $j=2$ make only a negligible contribution; the two expansion coefficients of interest are thus $k_{1}=(\partial k / \partial \omega)_{\omega 0}$ and $k_{2}=\left(\partial^{2} k / \partial \omega^{2}\right)_{\omega 0}$, which parameterize the (inverse) group velocity and (inverse) groupvelocity dispersion, respectively. By Fourier transforming back to the time domain, it can be shown that $A$ must satisfy

$$
\frac{1}{2 k_{0}} \frac{\partial^{2} A}{\partial z^{2}}+i\left(\frac{\partial A}{\partial z}+k_{1} \frac{\partial A}{\partial t}\right)-k_{2} \frac{\partial^{2} A}{\partial t^{2}}+\gamma|A|^{2} A=0 .
$$

where the coefficient of the nonlinear term is $\gamma=n_{2} / 2 n_{0}$. At this juncture, one should recognize that the double- $z$ derivative, $\partial^{2} A / \partial z^{2}$, appears naturally in the governing equation [5]. It is this term that is routinely neglected in analyses of pulse propagation phenomena.

Recently, it has been shown for the first time that spatial dispersion in some semiconductor materials can provide a second contribution to the coefficient of $\partial^{2} A / \partial z^{2}$ [4]. This field-exciton coupling augments the propagation contribution $1 / 2 k_{0}$ to yield a lumped coefficient, 


$$
\frac{1}{2 k_{0}}+\frac{n_{0} \Gamma \Delta \tilde{\omega}_{0}}{2 \delta \omega^{2} c}
$$

Here, $\Gamma \equiv \hbar / 2 M_{x}^{*}, M_{x}^{*}$ is the effective exciton mass, $\tilde{\omega}_{0}$ is a resonant frequency, $\Delta$ is a dimensionless parameter related to the oscillator strength for the coherent exciton-photon interaction, and $\delta \omega$ is a frequency detuning). A salient point is that the coefficient of $\partial^{2} A / \partial z^{2}$ can, in principle, become negative when $M_{x}^{*}<0$ [4].

After rescaling, the following governing equation for the dimensionless envelope $u$ may be derived:

$$
\kappa \frac{\partial^{2} u}{\partial \zeta^{2}}+i\left(\frac{\partial u}{\partial \zeta}+\alpha \frac{\partial u}{\partial \tau}\right)+\frac{s}{2} \frac{\partial^{2} u}{\partial \tau^{2}}+|u|^{2} u=0 .
$$

The normalized space and time coordinates are $\zeta=z / L$ and $\tau=$ $t / t_{p}$, respectively, where $t_{p}$ is the duration of a reference pulse and $L=t_{p}^{2} /\left|k_{2}\right|$. The sign of the group velocity dispersion is flagged by $s= \pm 1=-\operatorname{sgn}\left(k_{2}\right) \quad(+1$ for anomalous; -1 for normal), and $\alpha \equiv k_{1} t_{p} /\left|k_{2}\right|$. The spatial dispersion parameter is $\kappa$ $=\kappa_{0}+D$, where $\kappa_{0} \equiv 1 / 2 k_{0} L=c\left|k_{2}\right| / 2 n_{0} \omega_{0} t_{p}{ }^{2}$ and $D \equiv$ $n_{0} \Gamma \Delta \omega_{0} / 2 \delta \omega^{2} c L=\left|k_{2}\right| n_{0} \Gamma \Delta \omega_{0} / 2 \delta \omega^{2} c t_{p}^{2}$. Finally, $u=A / A_{0}$, where $A_{0}=(\gamma L)^{-1 / 2}=\left(2 n_{0}\left|k_{2}\right| / n_{2} t_{p}^{2}\right)^{1 / 2}$.

\section{MODELS IN THE LOCAL TIME FRAME}

When considering problems involving pulse propagation, one typically follows a prescribed route to get from the more general nonlinear-Helmholtz governing equation (6) to the more straightforward nonlinear-Schrödinger (NLS) model. Firstly, one typically invokes the SVEA by arguing that the term in $\partial^{2} u / \partial \zeta^{2}$ is small. A Galilean boost to a frame moving at the group velocity $1 / \alpha$ is then implemented by defining

$$
\tau_{\mathrm{loc}}=\tau-\alpha \zeta \quad \text { and } \quad \zeta_{\mathrm{loc}}=\zeta .
$$

The coordinates $\left(\tau_{\text {loc }}, \zeta_{\text {loc }}\right)$ are typically referred to as the "local time frame". They define a unique frame of reference in which a pulse centered on the carrier frequency $\omega_{0}$ is at rest. In this rest frame, the field $u$ satisfies the familiar NLS equation with a cubic nonlinearity,

$$
i \frac{\partial u}{\partial \zeta_{\mathrm{loc}}}+\frac{s}{2} \frac{\partial^{2} u}{\partial \tau_{\mathrm{loc}}^{2}}+|u|^{2} u=0
$$

for which many different types of solution are known.

The natural question to ask is, "what happens if one keeps the $\partial^{2} u / \partial \zeta^{2}$ term in Eq. (6) when implementing the Galilean boost?" In that case, the governing equation takes on a crossderivative operator:

$$
\begin{aligned}
\kappa \frac{\partial^{2} u}{\partial \zeta_{\mathrm{loc}}^{2}}+i \frac{\partial u}{\partial \zeta_{\mathrm{loc}}}+ & \frac{1}{2}\left(s+2 \kappa \alpha^{2}\right) \frac{\partial^{2} u}{\partial \tau_{\mathrm{loc}}^{2}} \\
& -2 \kappa \alpha \frac{\partial^{2} u}{\partial \zeta_{\mathrm{loc}} \partial \tau_{\mathrm{loc}}}+|u|^{2} u=0 .
\end{aligned}
$$

To proceed, one might, for instance, consider only those families of solutions where $\kappa \alpha^{2}<<O(1)$, which enables the coefficient of the temporal dispersion term to be simply $s / 2$. One could also present a case, based on order-of-magnitude considerations, for omitting the cross-derivative term. In so doing, one ends up with

$$
\kappa \frac{\partial^{2} u}{\partial \zeta_{\mathrm{loc}}^{2}}+i \frac{\partial u}{\partial \zeta_{\mathrm{loc}}}+\frac{s}{2} \frac{\partial^{2} u}{\partial \tau_{\mathrm{loc}}^{2}}+|u|^{2} u=0 .
$$

Biancalana and Creatore have recently analysed model (10) [4], which is a temporal analogue of the well-known spatial nonlinear Helmholtz equation [6,7]. They performed a linear stability analysis [8] of its continuous-wave solutions, and derived exact analytical bright and dark solitons. The work of Biancalana and Creatore [4] is particularly important since it appears to be the first inclusion of Helmholtz-type effects in scalar pulse modelling.

\section{THE LABORATORY FRAME}

Despite the excellent progress made with model (10), the above approximations to eliminate inconvenient terms is not always going to be a fully satisfactory approach [4]. For instance, the mapping between normalized and dimensional quantities is no longer exact. With this in mind, it is instructive to compare how the mathematical structure of the two simplified models differ from the envelope equation in the laboratory frame - Eq. (8) drops two terms $\left(i \alpha \partial u / \partial \tau\right.$ and $\left.\kappa \partial^{2} u / \partial \zeta^{2}\right)$, while Eq. (10) drops only a single term $(i \alpha \partial u / \partial \tau)$. Importantly, both simplified models admit exact analytical solutions, and this is undoubtedly one of the reasons for their success.

The nub of the problem is that if one wishes to keep the $\kappa \partial^{2} u / \partial \zeta^{2}$ term (which is essential, for instance, when describing spatial dispersion [4]), then Galilean boost (7) results in a local governing equation that is more complicated than the original equation! So at the outset, the conventional coordinate transformation serves no useful purpose. This leaves one with a fairly stark choice. One could either work with the approximate models (8) and (10), with all their inherent advantages and disadvantages; or one can abandon the nearuniversal Galilean transformation and remain in the laboratory frame.

It turns out that, if the latter option is chosen, a huge amount of progress can be made. So much, in fact, that it is surprising that analyses of optical pulses in the laboratory frame (i.e., in the frame where experiments are always performed and measurements made) seem to be almost completely absent from the literature. Rather than seeing how many terms can be dropped from Eq. (6), one can instead approach the problem in the reverse sense: Eq. (6) contains just one extra term compared to the spatial Helmholtz equation. This term even has a particularly tractable form - a linear operator $i \alpha \partial u / \partial \tau$ (i.e., there are no higher-order or cross-derivatives, and no complicated nonlinearities to consider). Hence, one expects to be able to deploy the same mathematical methods [6-8] and computational [9] tools that have been used extensively over the past decade to study Helmholtz spatial solitons. 

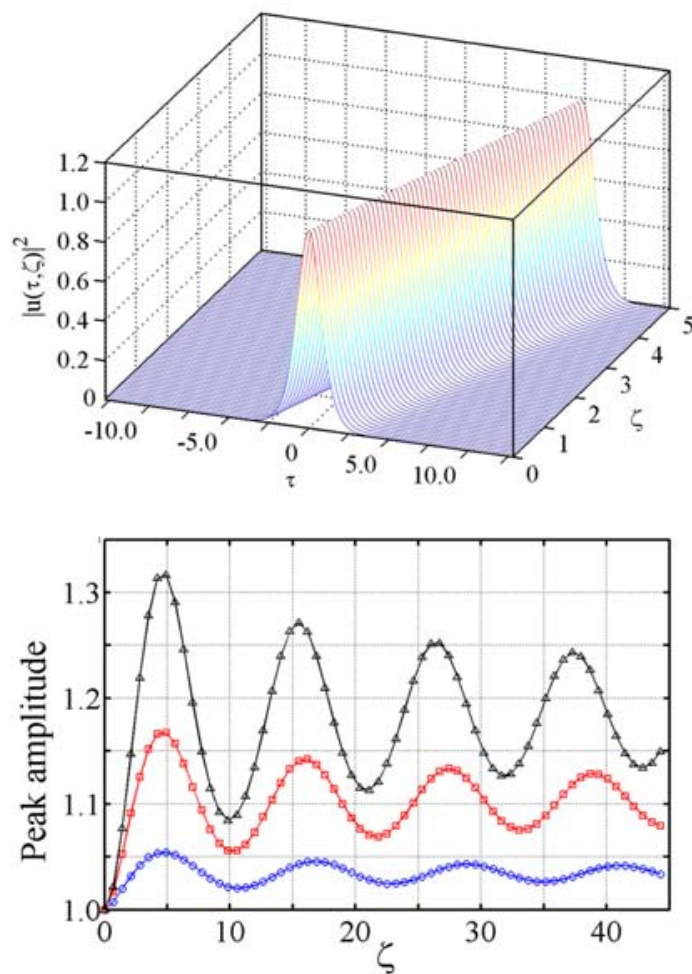

Fig. 1. Top: propagation of an exact bright soliton of Eq. (6). Bottom: typical self-reshaping oscillations for a perturbed bright soliton with $\kappa<0$.

\section{HELMHOLTZ SOLITON PULSES}

In this presentation, we will give a comprehensive overview of our investigations into Eq. (6). This will include a linear stability analysis of the continuous-wave solutions, and establishing where those solutions are modulationally stable against both long- and short-wave periodic perturbations.

Exact analytical bright and dark soliton solutions will also be reported, and their space-time geometry considered in detail. New parameter regimes will be considered that have no counterpart in the spatial domain - namely, $\kappa<0$ (in the spatial domain, $\kappa$ is the nonparaxial parameter, which must be positive $[4,6,7])$. A wide range of generic features will also be identified. Crucially, the properties of these new Helmholtz temporal solitons are found to depend on the sign of the product $s \kappa$, rather than $\operatorname{sgn}(s)$ or $\operatorname{sgn}(\kappa)$ separately.

One of the key results - and also one of the most interesting - is the velocity combination rule for Helmholtz solitons. This law, which is geometric in nature, is strongly reminiscent of the way velocities add together in relativistic particle mechanics. In fact, when $\operatorname{sgn}(s \kappa)=-1$, there is a one-to-one mapping with special relativity theory. Deeper insight can be gained by considering the transformation laws for Eq. (6), which show that the velocity combination rule is an intrinsic property of the model itself, rather than a property of particular (e.g., soliton) solutions.

Analysis has also uncovered why the Galilean transformation (7) is inherently incompatible with the Helmholtz governing equation (6). In conventional pulse theory, there exists a single unique reference frame where all pulses (centred on the carrier frequency $\omega_{0}$ ) are stationary. No such frame exists for the solutions of Eq. (6) because the transformation to the rest frame of any pulse is parameterized by the characteristics (e.g., the intensity) of the pulse itself.

Finally, we will provide supporting evidence of the stability of the new soliton pulses that has been obtained from extensive computer simulations [9]. For example, bright solitons subjected to an initial perturbation to their local temporal shape tend to exhibit monotonically-vanishing oscillations, relaxing back toward an invariant solution (see figure 1).

\section{CONCLUSION}

We have taken the first steps toward understanding nonlinear optical pulses from a new perspective by studying their behaviour from the laboratory frame. In the course of our work, we have found that this frame is the natural frame from which to describe pulses. Furthermore, the internal inconsistencies introduced by the classic Galilean boost (7) can be quite subtle (indeed, some of them remained hidden until the more general Helmholtz model was investigated). We have discovered, what we believe to be, a compact and elegant framework for describing optical pulses. The framework is exact [in the sense that no further approximation beyond Eq. (6) is required] and self-consistent. The existence of bright and dark soliton solutions with novel relavitistic characteristics is also reported.

\section{REFERENCES}

[1] A. Hasegawa and F. Tappert, "Transmission of stationary nonlinear optical pulses in dispersive dielectric fibres. I. Anomalous dispersion," Appl. Phys. Lett. vol. 23, no. 3, pp. 142-144, 1973.

[2] L. F. Mollenauer, R. H. Stolen, J. P. Gordon, and W. J. Tomlinson, "Extreme picosecond pulse narrowing by means of soliton effect in single-mode optical fibers," Opt. Lett. vol. 8, no. 5, pp. 289-291, 1983.

[3] V. M. Agranovich and V. L. Ginzburg, Crystal Optics with Sptial Dispersion, and Excitons, Berlin, Springer, 1984.

[4] F. Biancalana and C. Creatore, "Instabilities and solitons in systems with spatiotemporal dispersion," Opt. Exp. vol. 16, pp. 14882-14893, 2008.

[5] K. J. Blow and N. J. Doran, "Solitons in optical fibres," in Nonlinear Waves in Solid State Physics A. D. Boardman et al. Eds. Plenum 1990, pp. 325-369.

[6] P. Chamorro-Posada, G. S. McDonald, and G. H.C. New, "Nonparaxial solitons," J. Mod. Opt. Vol. 45, no. 6, pp. 111-1121, 1998.

[7] P. Chamorro-Posada and G. S. McDonald, "Helmholtz dark solitons," Opt. Lett. vol. 28, no. 10, pp. 825-827, 2003.

[8] J. M. Christian, G. S. McDonald, and P. ChamorroPosada, "Helmholtz bright and boundary solitons," J. Phys. A: Math. Theor. vol. 40, pp. 1545-1560, 2007.

[9] P. Chamorro-Posada, G. S. McDonald, and G. H. C. New, "Non-paraxial beam propagation methods," Opt. Commun. vol. 192, pp. 1-12, 2001. 